\title{
myCourse: An Educational Content Management Platform with Social Networking Characteristics
}

\author{
$\underline{\text { doi:10.3991/ijet.v5i4.1450 }}$ \\ T. Giouvanakis ${ }^{1,2}$, G. Fragidis ${ }^{1}$, I. Ignatiadis ${ }^{2}$ and A.Koumpis ${ }^{2}$ \\ ${ }^{1}$ Institute of Technology and Education, Serres, Greece \\ ${ }^{2}$ Research Division, ALTEC Software, Thessaloniki, Greece
}

\begin{abstract}
We adopt the direction towards new educational and pedagogic paradigms, where learning is a process of emergence and co-evolution of the individual, the social group and the wider society. In this direction, ServiceOriented Architectures are becoming a popular system paradigm for e-learning. In this article, we present our research and development efforts to provide a social networking learning platform for developing services which address the personal learning needs of the users and enable them to create value. We also present the specific characteristics of our community driven service framework and discuss our approach in comparison to other similar approaches and frameworks.
\end{abstract}

Index Terms-User Generated Content, Social networking, Educational services, Service Oriented Architectures.

\section{INTRODUCTION}

Everybody accepts the enormous success of many social networking services which meet personal needs of friendship (Facebook), concise text-based communication (Twitter), professional networking (LinkedIn), bookmarking (delicious) and sharing videos (YouTube) and pictures (Flickr). The success of these services rests on the fact that the user/client/consumer is able to actively participate in a manner which is of personal benefit to the user, thereby creating value for himself/herself.

The idea that motivated us to proceed with the implementation of the myCourse environment has been to follow on the success of the aforementioned services and create additional value for the individual learner and the wider community, in terms of facilitating the creation, mashing-up, publishing, tagging, using and rating learning services in a simple and user-friendly interface. Whereas Flickr concentrates on pictures and YouTube concentrates on videos, myCourse concentrate on (web) services created for and by users within a given social networking environment of a structured learners' community (e.g. high school or MBA class, etc.).

In this respect, the aim of myCourse is to provide a social networking platform for developing services which address the personal learning needs of the users and enable them to create value. More specifically, myCourse covers two main types of users using the educational service platform:

1. Novice inexperienced users without any technical knowledge or programming expertise. (This first group might be regarded as the 'students', though this classification in the context of myCourse is not holding for reasons we show later in the paper.)
2. Professional users wanting to promote their own services, either for free or via a chargeback mechanism. (Again, this second group could be regarded as the 'teachers', though, same as above, this classification is not correct.)

The myCourse Framework (consisting of the myCourse platform, the associated service and business models, as well as relevant tools and technologies) aims to:

1. Help learners create highly individualised (web) services to meet their needs, whether for meeting educational, business or self-development purposes

2. Dynamically integrate people's multidimensional profiles

3. Provide a roadmap for creating social networking services to support educational service provision

4. Exploit the wisdom of the crowds (crowdsourcing) in providing better educational services, while at the same time promoting open innovation and enhancements for educational service providers.

5. Versatility is a central attribute of the myCourse framework, enabling the consumption of services which address needs in a complex and highly distributed networked environment which can be seamlessly integrated with the current web.

The objective is for our framework to be a major driver for large scale exploitation, enabling users to create, share and configure services easily which address theirs and others' needs, whether for entertainment, information, business or any other area.

Our efforts till today have concentrated on the implementation of a prototype to incorporate two use cases (via provision of web services) in the areas of finance and tourism respectively.

\section{A. Basics of myCourse environment}

myCourse can be defined as a framework for educational service provision based on learners' needs. This requires a well defined concept of service which we currently define it as: "service is any item or action someone can offer which could meet the needs of that or another person". The Framework is need-driven from the user aspect in order to provide 'made to fit' personalized services - rather than non- customised 'one-size fits all' solutions.

The concept of the myCourse Framework is depicted in Figure 1. This shows that a person with a particular learning need can use a solution from a network of people and services. The evolution, expansion and healthy functioning of this network are dependent on the ability of indi- 


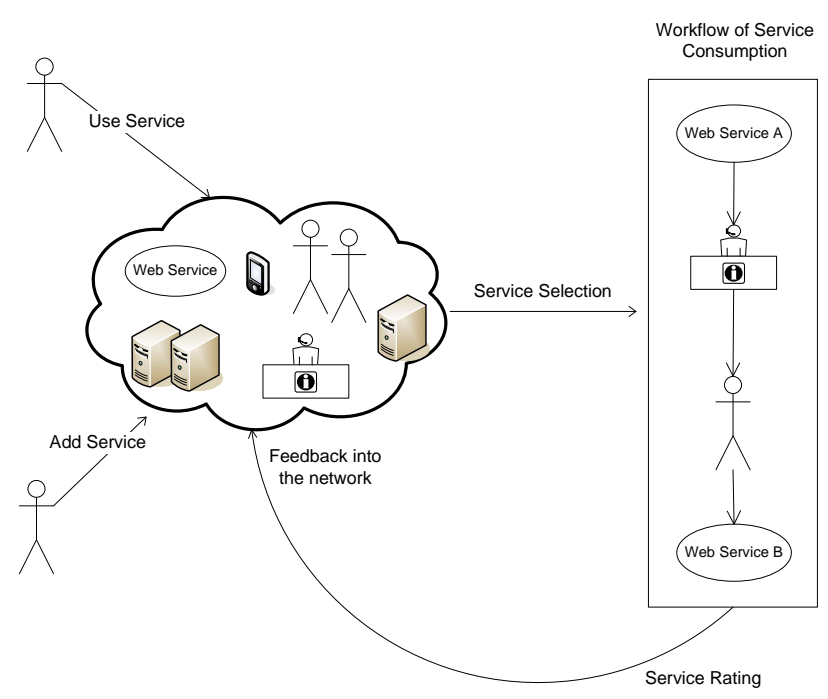

Figure 1. myCourse Service Framework

viduals or organizations to add their services to the network. The functions would be complemented by feedback and rating of utilized services (ultimately promoting open innovation and enhancing service quality) as illustrated in the Figure.

Such a framework enables the implementation of social networking services which support both networking for their users (as does Facebook) and the provision of educational services by individuals or organizations. These services can also be networked and mashed-up. Mashingup services, i.e. combining data or functionality from two or more external sources to create a new service, in a social network fashion could assist the selection and composition of services to address complex social and business needs.

People are multidimensional and multifaceted and defining the range of these complex aspects would be necessary in order to enable to locate and use the appropriate educational services. Personal profiles and service tagging should vary from short and simple to complex and detailed.

myCourse provides a formalized structure for supporting service discovery, selection and composition (mashing-up), together with the relevant social networking functions (tagging, blogging, reviewing and rating).

Research into the categorization of services is essential. This includes the division into the individual's personal needs and the needs of an organization. Geographical location, language and other such topical considerations need to be additionally taken into account.

The idea of a framework for such a type of highly (inter-)networked services which is open to use by anyone in practice may seem too ambitious and easy to compromise, however the wisdom of the crowds in social networking services has helped to override such problems (e.g. in Wikipedia where false information is quickly corrected by others or a warning is provided regarding its reliability). Such approaches will ensure the quality and monitoring of level of service.

myCourse provide also suggestions for the consumption of services (see Workflow of Service Consumption in Figure 2). For this, research has been conducted into dif-
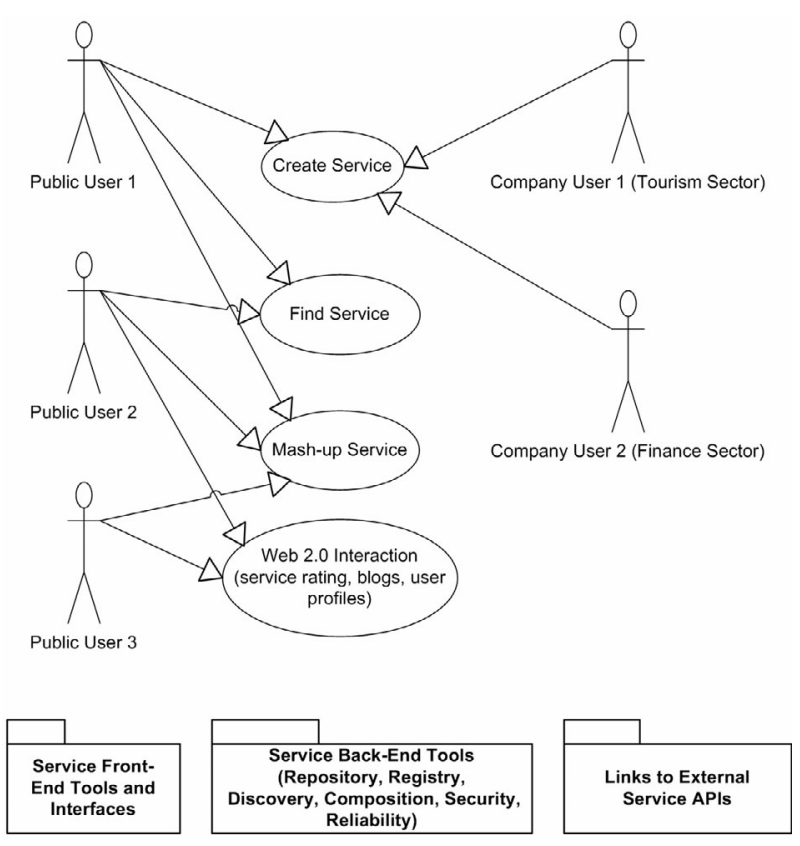

Figure 2. myCourse main use cases

ferent types of services which already exist. A service can be software or non-software related. It can also be a combination of both. myCourse, again, provides individuals or organizations with the appropriate combination of these services. For this purpose the use of workflow engines (as part of services provided by organizations within the platform) which can be dynamically configured during service composition and also edited by a user has been examined. The appropriate service/combination of services also needs Service Level Agreements between the provider and consumer, especially in the provision of business (as opposed to user) services.

From the above, it is easy to see that myCourse may easily become a roadmap for developing open serviceprovision services of the Web 2.0 genre, maintaining enough versatility to eventually address provision of needs in the upcoming Web 3.0, where semantic considerations are of importance. A main part of our currently undergoing research work relates with the development of an open architecture that will allow in the near future incorporation of semantic functionalities where needed.

The figure below illustrates the main (high-level) use case of the platform developed as part of the myCourse functionality when applied in capacity building projects for professional users (public administrations or corporate users in the world of enterprises in the tourism and finance sectors).

The next section presents the test application scenarios that will be developed as part of the project and which will be included in the platform (Social Network Site) as demonstration (business) services.

\section{MYCOURSE ARCHITECTURE}

The conceptual architecture of myCourse is comprised of the components Profile, Relation and Content \& App (Service). These are as follows:

Profile: Visitors can just browse the common contents that the socialized business network provides such as news, learners material and advertisement, etc. If they want to leverage more services based on the platform, 
such as create, customize, rate or mash-up B2B, B2C, C2C services, have blog, library folders, video etc., they

have to register as a member. If they further want to create a professional business based on their services and exploit additional functionality such as chargebacks, SLAs, QoS, marketing etc, they have to register as business users.

Relation: Provide visibility, connectivity and interactive service among myCourse users within the myCourse community of users by good friend list, messages within community, access to each others' home pages, etc. This will facilitate user to user assistance, share best practices and form new interest groups by their (personal and business) preferences, etc. Keep user fidelity and loyalty by different kind of themes such as peers, fans, enthusiasts, etc. Leverage the strong relationship within the Social Network structure such as that of the friends, exclassmates and peers etc. to establish, enlarge, enforce and maintenance the relationships between service producers and consumers. Leverage rating and comment mechanism to elevate one's credit. Leverage News Feeds to spread and update dynamic information among the relation chains and groups. News Feeds also act as an effective marketing method for users with business propose because it's more believable among friends and familiars.

\section{Content \& App ( Service ):}

There are two kinds of content:

1. The content provided by the Social Network Services (SNS) such as news, learning content and advertisement, etc.

2. User Generated Content (UGC) includes user's blog, user provided learning material in the form of text, audio or video, ratings, etc. The major part of the myCourse content consists of UGC.

App (Service) includes web and mobile application, SaaS and SOA services, as facilitated by the relevant tools within the platform.

The social network users or organizations may establish their own C2C, B2C and B2B business as facilitated by the relevant (user-defined) services. In the tourism case for example, it will seamlessly integrate the upstream and downstream industries of the tourism ecosystem by leveraging the "third party generated services" such as traffic and resort ticket booking, hotel and restaurant reserving, e-paying, tour guide service and location-based services, etc. Users may get their answers in discussion with friends and with Wiki.

This SNS serves as an open and dependable service platform eventually allowing individuals and organizations users to establish their own business and leverage the relationships there to promote their commodities and services. Scalability of the platform will be considered in the architecture design. Service Level Agreement (SLA) management, Quality of Service (QoS), access rights and customer charging will be supported for the case of business services, as will semantic interoperability (where appropriate) and access to educational service repositories.

\section{A. myCourse technical architecture}

The technical architecture of the myCourse platform includes 3 layers:

1. Service Front Ends: a set of user-friendly, graphical tools to allow users to create, search, configure and use services, including Web 2.0 functionalities to fa-

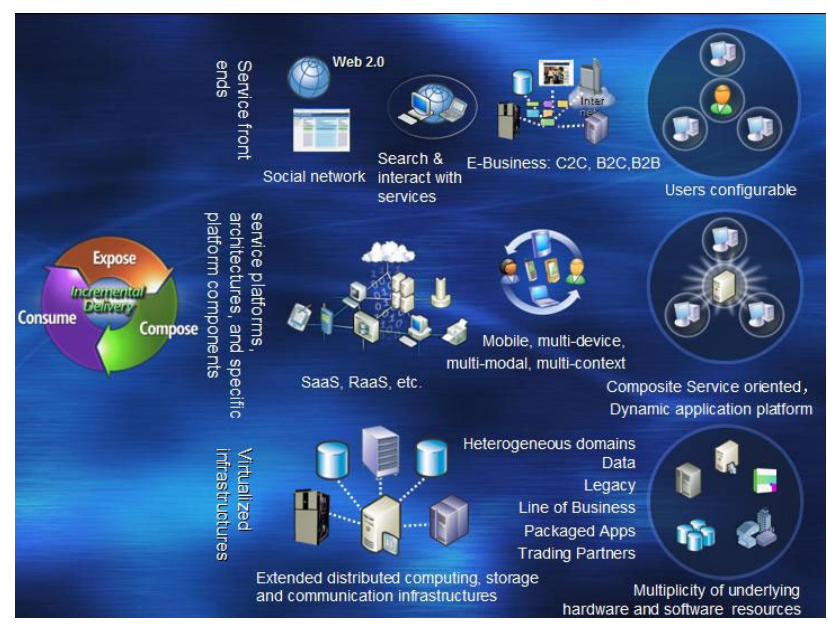

Figure 3. myCourse Technical Architecture Layers

cilitate a social network centred on services and user profiles and business considerations such as C2C, B2C and B2B for business-registered users.

2. Service platform and components developed so that they can be used externally and in other environments. Mobile, multi-modal and multi-context implementations are considered according to user needs and accessibility capabilities (not all high school boys and girls can afford to have a i-phone and a fast Internet connection!...). Service publishing, discovery and composition is also facilitated.

3. Virtualized infrastructures: these are considered as the appropriate storage and communications infrastructure for use in heterogeneous domains, while also considering non-functional requirements such as security, reliability, extendibility and resiliency.

It is easy to see that with myCourse we do not reinvent the wheel; synergies with regards to technical considerations (architecture, virtualised infrastructure) as well as service considerations (front-ends, engineering) have been explored, in order to capitalize on the research results of these projects. myCourse adds the very important aspect of the Social Networking, and the different Service and Business Models within the Technical Architecture of the myCourse platform.

\section{MYCOURSE RELATION With OTHER APPROACHES}

In this section we carry out a brief literature review on topics relevant to our approach, so we can pinpoint the progress that is made beyond the current state of the art.

\section{A. Web 2.0 and Social Networking Sites}

Over the last few years, the rise of social software and social networking has driven the transition from contentcentred to people-centred activities. 'Reading' and top down content production has dominated the first era of the Internet. Now a truly participatory bottom up or 'read/write' approach is emerging as a dominant future trend. This shift of focus represents as much a cultural phenomenon as a technological one and has resulted in many commentators questioning its impact on our sense of identity, the meaning of community and the nature of this change (Hatzipanagos \& Warburton, 2009).

Web 2.0 has moved beyond the original meaning of the term defined by O’ Reilly (2005) to encompass a set of tendencies exhibited by virtual communities. According to 
O’ Reilly (in Musser, 2006), Web 2.0 is a set of economic, social and technology trends that are based on user participation, openness and network effects. It is beyond doubt that consumer-oriented concepts can easily grow in such an environment. For instance, the best-known success stories of Web 2.0 (e.g. Wikipedia, Facebook, YouTube, etc.) are based on the concept of user participation through social networking.

The terms Web 2.0 and social software and social networking are often used together or synonymously, though Web 2.0 describes more the new ways in which the WWW is used, while social software and social networking, built on Web2.0 platforms and services, describes the universe of possible interactions between individuals and communities, where users are connected and collaborate with each other. The attitudes and behaviours of these communities or social groups have become as significant as the distributed technological platforms that are being exploited by increasing numbers of internet users.

One of the major characteristics of these new practices has been a shift towards 'user-generated content' where:

- Collective and collaborative information is gathered, shared, modified and redistributed in creative acts;

- Personal sites and content increasingly belong to the so called 'me media' category;

- The user controls the choice of appropriate software, tools and services;

- The 'collective intelligence' of users is harnessed through aggregation and large-scale cooperative activities (O’ Hear 2005).

For example, social network sites, have become integrated into the daily practice of millions of users and Boyd \& Ellison (2007) describe the key features of these services as allowing individuals to:

- $\quad$ construct a public or semi public profile within a bounded system

- $\quad$ articulate a list of other users with whom they share a connection, and

- view and traverse their list of connections and those made by others within the system.

Turkelang has identified two important functions of these social networks:

- Intelligence gathering, where people share and construct information online

- $\quad$ Meeting new people, where there are opportunities for one to one and one to many and many to many interactions.

Participants in internet-based social networking are immersed in fragmented digital environments, and engage in acts of computer mediated communication (Hatzipanagos 2006) through e-mail, email-conferencing and mobile texting, podcasting, personal publishing via blogs and wikis, aggregation and mash-ups, voice, chat, instant messaging and videoconferencing. Social networking is productive of and exercised by virtual communities of people with common interests. Users have the opportunity to contribute to personal and informal or professionally oriented social networks and the goal can be employment, provision of a service or collaboration.
The term community has been expanded to include interdependency and a set of relationships that connect people and groups. Citizens become Netizens, an identity that relates them to the entire world, and moves them outside their local life and work settings. For example, social networking, such as Facebook, MySpace, SecondLife and LinkedIn have changed forever the way people communicate in formal (professional) or informal spaces.

In all these cases, instead of business-generated content we see user-generated content; the users contribute directly or indirectly and collectively co-create content or experiences. The users are not only consumers, but also co-developers; they do not expect the passive fulfilment of their needs by business firms rather, they participate actively in the development of products and services that meet their needs. Their motives for participation are related to their needs to create products, services and websites that fulfil their personal interests, to tailor offers according to their preferences, to experiment, learn and gain experiences, to contribute to the community, to offer to their peers and to communicate and share with others.

\section{B. Consumer oriented concepts in business}

We can distinguish three approaches on value creation: a) the production-oriented approach, b) the marketingoriented approach and c) the consumer-oriented approach.

The production-oriented approach conveys the traditional ideas of the manufacturing paradigm and expresses the notion of "value-in-production" where emphasis is put on the role of the producer.

The marketing-oriented approach is an extension of the production-oriented approach that introduces the notion of "value-in-dissemination" and draws attention to the role of marketing in organisations as a means for the creation and dissemination of value. The marketing mix is the key instrument of this approach,

The consumer-oriented approach introduces the notion of "value-in-use" by suggesting that value is created only when products or services are used by the consumer. The basic assumption here is that the consumers, not the business firms, have value-adding activities (Normann \& Ramirez, 1993) and activity chains (Sawhney et al., 2003) and that the business processes create the pre-conditions for the creation of value. Value is defined by the consumer during the selection of offerings, i.e. products or services, and created by the consumer during the consumption of the offerings.

According to Vargo and Lusch (2004), a firm can only make value propositions and then, if the proposition is accepted, co-create value with the consumer. Therefore, the consumer becomes a "co-creator of value".

The concept that the consumer participates in the creation of value is well established in the literature (e.g. Normann \& Ramirez, 1993; Prahalad \& Ramaswamy, 2004; Zuboff \& Maxmin, 2004; Von Hippel, 2006). Lusch et al. (2007) distinguish between value co-creation and value co-production; the former refers to the determinant and catalytic role of the consumer in the creation of value, while the latter refers to the supportive role of the consumer in the execution of business processes (e.g. in self-service settings, in design processes, in finishing products or services, etc.). Customer participation and coproduction schemes are popular in the context of businessto-business collaboration; however, they are still a grey 
area in the relationships with the consumer and a question mark whether they truly serve the consumers needs.

From the above it is easy to see that myCourse supports definitely follows a consumer-oriented approach.

Communication and dialogue are essential to the consumer-oriented approach. The approach in value creation generates a new situation for businesses and other service providers affecting their relationships with the consumer. Some authors describe the new situation as the "relationship economy" or the "support economy" (Zuboff \& Maxmin, 2004), with markets being transformed into "forums" (Prahalad \& Ramaswamy, 2000), in which consumers can enter into dialogue about their needs with business firms and peers and synthesize individualized solutions that fulfil their needs.

myCourse supports dialogue through social networking while allowing individuals as well as businesses to partake in service provision as well as businesses.

Service-Dominant Logic has been recognized as a conceptual foundation for a service-based economy, as well as for the development of service science (Lusch et al. 2007, Maglio \& Spohrer, 2008, Spohrer et al., 2008). Service is defined here as the application of specialized competences (knowledge and skills) for the benefit of another entity (Vargo \& Lusch, 2004). Hence, the fundamental unit of exchange in all cases is the application of specialized skills and knowledge, despite the fact that the embodiment of knowledge in tangible outputs creates the deceptive distinction between products and services. In essence, all economies are service economies, because the exchange of knowledge and skills characterizes all economic activities (Vargo \& Lusch, 2004).

According to the Service-Dominant Logic, value creation is based on service provision. Since the benefit from the service provision is manifested in the context of the customer, it means that what firms provide should not be understood in terms of outputs with value, but rather as inputs for a continuing value-creation process with and by the consumers.

If the consumer-oriented concepts for value creation are right, then business firms should reconsider their business models according to the concept of service and they should try to identify how to innovate with services and co-create value with the consumer. This is, in general, the purpose of service science (Chesbrough \& Spohrer, 2006).

This is also a purpose of myCourse. Much of myCourse Services (and especially the test scenarios in the areas of finance, tourism and employment) draw from the emerging Service Science which is conceived as a multidisciplinary effort to understand the nature of services, how they should be designed, produced and delivered and how to innovate in a service-based economy.

\section{Consumer-oriented concepts in e-services and business networks}

Information and communication technologies and the Internet have great significance in a service-based economy.

Just as service is not clearly defined in the literature, so too is the term e-service. Rust \& Lemon (2001) consider that the term is used in general to denote transactions in which information is the primary value exchanged.
Gronroos et al. (2000) claim that e-service is any product or service that is exchanged over the Internet. Others restrict their scope on services that are delivered electronically or over electronic networks (Rust \& Kannan, 2003). In myCourse, the focus has been put on any type of (web) service that can be found or exchanged via the Internet.

The Internet tends to shift bargaining power to end consumers in their transactions with businesses because it allows the end consumer to get in contact directly with a great number of producers. On the other hand, the concepts of "mass customisation" (Gilmore \& Pine 2000), "one-to-one marketing" and "long tail economics" (Anderson, 2006) are based on the premise that, with the support of information technologies, business firms are able to target each consumer separately, personalise their services and disseminate them efficiently.

This has been be taken into account in myCourse; the Framework supports both 'one-to-one' and 'many-tomany' marketing and dialogues within a social network which enables the consumer to communicate with providers and other consumers able and willing to meet a need.

In order to design this network of consumers and providers ideas can be gleaned from current business networks and ecosystems. In the literature we can find different kinds of business networks, such as business constellations (Normann \& Ramirez, 1993), extended enterprises (Prahalad \& Ramaswamy, 2003), value nets (Bovet \& Martha, 2000), virtual enterprises (Sawhney \& Parikh, 2001; Walters \& Lancaster, 1999), strategic networks (Jarillo, 1988) and business ecosystems (Moore, 1996; Iansity \& Levien, 2004). The concept of business ecosystem is a metaphor that steps forward the movement towards symbiotic and co-evolutionary business networks.

Although myCourse allows user-defined services as well, in the case of business services, myCourse network takes the form of a business and consumer ecosystem. Here, the role of the learner is of prime importance. Therefore, the network is crafted to respond to his/her special needs and where these needs cannot be met the social network would rise to fill this gap. This would also be of use in cases where the services sought are not related to business services, but rather of a voluntary nature.

\section{The consumer-oriented potential of Service-Oriented} Architectures and Mash-Ups

The OASIS Group defines Service Oriented Architecture (SOA) as a powerful framework for matching needs and capabilities and for combining capabilities to address those needs (OASIS, 2006). Services in SOA are defined in a similar way to the definition of service in the business world that is as deeds performed by the service provider for the benefit of the service client. Consequently, from a conceptual point of view, SOA could be used to provide the technological foundations that are required for the empowerment of consumers in the selection, composition and consumption of products or services in electronic markets.

The services' world is discussing issues more closely related to providing Software as a Service (SaaS) components and how we can handle issues relating to security and transactions in web services. Security, the first of these issues, allows for secure usage of services regardless of the underlying platform and provides specifications (like WS-Security - Rosenberg et al. (2004)) and lan- 
guages (SAML - OASIS (2009)) for secure services. Additionally proposals for supporting transactions in web services have already been implemented, like the WSAtomicTransaction - OASIS (2009)). Finally, the Software as a Service (SaaS) model promises to deliver "all” existing software that we use daily on our computers as services in the near future - this idea is now slowly emerging and it has being exploited in the design of myCourse.

From an operational point of view, a SOA can be implemented with the use of Web Services. The basic Web Services model endorses three roles (service requestor, service provider and service registry) and three operations (publish, find and bind). Web Services follow the "find, bind and invoke" paradigm, where a service requestor performs dynamic service search by querying the service registry for a service; if the service exists, the registry provides the requestor with contact details for the service.

Such an operational model is clearly consumer-oriented and could support consumer-oriented value creation. The service requestor recognizes some need, searches for solutions, makes the selection, invokes the service and composes it with other services in his own context, in order to create value for him. Through a social network, other users can assist the service requestor in the composition and provide feedback.

Composition can be implemented with the use of mashup technologies. Mash-ups are a new kind of data intensive and data integration applications which are based on the fusion of heterogeneous data sources that provide a public set of APIs. Depending on the API either a more traditional server based content generation process is followed or a client side scripting language or applet is used to mash-up the content. Most of the available mashups rely on the first solution and use the browser side technologies to produce an aesthetically pleasing result for the user. A technology or better an application model that characterizes mash-ups is AJAX (Asynchronous Javascript and XML) Lauriat (2007). AJAX has revolutionized the way web applications behave and provide a more robust and fulfilling user experience.

Other technologies involved in developing and supporting mash-ups include web services related protocols like SOAP (W3C, 2009) and REST Tyagi (2006). SOAP is the basic message exchange protocol used by services (including of course services used in the mash-ups) to communicate with each other. On the other hand REST provides a simple protocol for web services that supports only basic functions (like POST, PUT, DELETE, etc) but its simplicity makes it a prime candidate for mash-ups since it allows the easy fusion of different data sources.

An important issue in the mash-ups is the interoperability among the different data sources. This is addressed by the use of semantic web technologies like RDF/S based ontologies and RDFa (W3C, 2009) based annotations. That way data and their meaning can be seamlessly exchanged among the different sources and can carry their real meaning along. Mash-ups can be used as the main integration point for semantically described data bringing closer the concepts of Semantic Web and Web 2.0 by providing the best of both worlds.

Composition of services through mash-ups is also a consumer-oriented approach, as the consumer decides which services to mash-up and in which way in order to create value for him/herself and the community at large.

It must also be mentioned however that most existing research on Service-Oriented Architectures focuses on software services which are seen as software components providing access to "real" services (for example a software service for travel booking provides access to the actually service for travelling) (Pistore et al., 2009). In addition, software services are used, but not necessarily owned (in the sense of being able to customize them according to needs) by consumers (NESSI, 2006). This leads to the needs to define Service Level Agreements where appropriate (e.g. in chargeback mechanisms) as software services are not used exclusively by their producers.

We recognize the importance of technical considerations (e.g. Service Level Agreements) for business services. However, we also aim to provide a more userfriendly approach to services, trying to contribute towards viewing the Internet as an enabler of "real” services aimed towards the end consumer, instead of pure "software" services created by the provider of these services.

As has been mentioned, there are two levels of users in the system, and for each of those different requirements are foreseen to be essential:

- For business users, they may want to sell their services, and therefore issues such as Service Level Agreements, Quality of Service, chargeback according to different business models are still important. These considerations point to looking at the technical aspects of web services, therefore we are still looking at services from a "software" point of view.

- For public users (who we assume to be not technically-savvy and want as a user-friendly interface as possible), "real-world" considerations of services are of importance. It is for these reasons that some key assets relating to services must be defined with which the user (consumer) is able to construct / mash-up / configure services. Following the paradigm by Pistore et al. (2009), these assets (which can be enablers or constraints) are:

1. Time, representing the temporal relation of the activities of the user, as well as conflicts and overlaps between these activities.

2. Location, representing the (current and perspective) location of the user, the availability of (real-world) services in these locations, as well as the necessity of moving or travelling to use these services.

3. Social relations (representing other parties such as family, friends, colleagues) involved in the user activities.

4. Money and other values, representing costs and assets involved in the user activities.

\section{E. Positioning with respect to other Service}

\section{Frameworks and research initiatives}

Several types of service frameworks exist which either focus in offering business services or focus on the technical side of service provision. Some examples of such frameworks are as follows:

- Balakrishnan (2000) described the e-speak Service Framework Specification for the concepts involved in implementing a totally inter-operable ecosystem of 
e-services encompassing both the business-tobusiness and business-to-consumer domains. These ecosystems integrate such things as supply chain automation, e-procurement, collaborative ecommerce, trading exchanges and personalized business-to-consumer interactions.

- The Intelligent Middleware Service Framework (Zongwei et al, 2006) is a service oriented framework that enables integrated e-logistics infrastructure and networks.

- The Follow-Me services framework (Kwak, 2007) provides connected ubiquitous services. The service session is preserved via the Session Initiation Protocol (SIP) even though an end-user moves from one computing environment to another. To get the follow-me services, all one has to do is to carry a small mobile pad which shows a list of devices that can be used as I/O devices of the on-going service session.

- García-Sánchez et al. (2009) propose an intelligent agent-based framework for the provision of semantic web services, integrating of the three technologies in order to offer services.

- Lamparter et al. (2006) illustrate a policy framework for trading configurable goods and services in open electronic markets. The framework allows the declarative description of seller pricing policies as well as buyer preferences over these configurations.

As can be seen from the above, a user- and consumeroriented framework based on web service creation and use per se does not currently exist, nor does a Social Networking Site based on these issues.

Though the work carried out in many of the abovementioned research projects is highly relevant to myCourse and synergies could be possibly exploited, however, none of these projects considers the social interaction aspects of services and the corresponding advantages.

Although we provide the technical infrastructure for an open, adaptable and scalable infrastructure, we did not reinvent the wheel by creating architectural and infrastructural concepts that are already exploited in other EU projects; we rather exploited valuable synergies with these projects. However we added the very important aspect of providing social networking facilities targeted towards the service consumer, whilst placing particular importance on the conceptualisation of services and the meeting of key individual, social and business needs.

\section{CONCLUSIONS}

The major impact of myCourse is in the contribution of high-added value educational services and the creation of online dynamic learners' communities (social networking) centred on service consumption and creation. This in turn leads to lowered barriers and new service and business models for service providers, while also strengthening the field of educational service provision, and contributing towards the future internet (the internet of services).

myCourse puts in place both a framework and an innovative service front end that results in learner empowerment in the context of online educational communities. myCourse users have the opportunity to contribute to personal and informal or professionally oriented social networks and the goal can be employment, provision of service or collaboration. Furthermore, amateur users of
myCourse may work together to utilise their collective experience and create consensus on good practice, going beyond facts by delving deeper into analysis and interpretation of information and services encountered in the Web.

Such behaviours and community activities can have an impact on:

- Collecting important 'user-generated content' which can inform educational services and individual learner needs

- collaborative work to gather information and evaluate, modify, share, and redistribute knowledge (achieved through social interactions such as service rating and feedback)

- harnessing 'collective intelligence' of learners where people share and construct educational content online through aggregation and large-scale cooperative activities (crowdsourcing)

- the construction of learners' public or semi public profiles within the myCourse system, to enable associations of learners via interests, needs and preferences

- Creating opportunities for one to one, one to many and many to many interactions.

- Combining educational web service creation and use with interaction and collaboration

It is the nature of the social media that allow this kind of affordances adding a people-centred aspect to the web services by enriching such online environments with activities, where users are connected and collaborate with each other. An interdisciplinary approach is required to engender this type of participation, crossing cultural boundaries and taking advantage of the enhanced opportunities such communities can offer. The possibility exists for a "massive" uptake of the results of myCourse by the industry.

The innovation of myCourse is that instead of businessgenerated content the users can contribute and collectively co-create content and share learning experiences. The users become 'prosumers', i.e. not just only passive consumers of educational content and learning services, but active providers and co-developers by participating actively in the development of learning products and services that meet their needs.

\section{REFERENCES}

[1] Anderson, C. (2006). The Long Tail: Why the Future of Business is Selling Less of More, Hyperion, ISBN 1401302378, New York.

[2] Balakrishnan, R. (2000). A Service Framework Specification for Dynamic e-Services Interaction. Fourth International Enterprise Distributed Object Computing Conference (EDOC'00) (pp. 2829). doi:10.1109/EDOC.2000.882341

[3] Bovet, D. \& Martha, J. (2000). Value nets: reinventing the rusty supply chain for competitive advantage, Strategy \& Leadership, Vol. 28, No 4, (pp. 21 - 26). doi:10.1108/10878570010378654

[4] Boyd, d. \& Ellison, N.B. (2007). Social Network Sites: Definition, History, and Scholarship.

[5] Cardoso, J., \& Sheth Amit P. (2006), Semantic Web services, Processes and applications, Springer, ISBN 978-0387302393. doi:10.1007/978-0-387-34685-4

[6] Chesbrough, H. \& Spohrer, J. (2006). “A Research Manifesto for Services Science" Paper presented at the Communications of the ACM, July 2006

[7] Gilmore, J.H. \& Pine, B.J. (2000). Markets of One: Creating Customer-Unique Value through Mass Customization, Harvard 
Business School Press, ISBN 978-1578512386, Boston, Massachusetts.

[8] Garcia-Sanchez, F., Valencia-Garcia, R., \& Martinez-Bejar R. \& Fernandez-Breis, J. (2009). An ontology intelligent agent-based framework for the provision of semantic web services. Expert Systems with Applications (pp. 3167-3187). doi:10.1016/j.eswa. $\underline{2008.01 .037}$

[9] Gronroos, C. (1990). Service management: A management focus for service competition, International Journal of Service Industry Management, Vol. 1, No. 1, (pp. 6-14). doi:10.1108/09 $\underline{564239010139125}$

[10] Hatzipanagos, S. \& Warburton, S. (2009). Social Software and Developing Community Ontologies (London: Information Science Reference, an imprint of IGI Global).

[11] Hatzipanagos, S. (2006). HOT and Flaming Spirals: Learning and Empathic Interfaces in Text-based Discussion Forum Dialogues. European Journal of Open, Distance and e-Learning, 2006/I.

[12] Iansiti, M. \& Levien, R. (2004). The Keystone Advantage: What the New Dynamics of Business Ecosystems Mean for Strategy, Innovation and Sustainability, Harvard Business School Press, ISBN 978-1591393078, Boston Massachusetts.

[13] Jarillo, J. C. (1988). On Strategic Networks, Strategic Management Journal, Vol. 9, No. 1, (pp. 31-41). doi:10.1002/smj. $\underline{4250090104}$

[14] Kwak, Y. (2007). Follow-Me services framework. Information and Communications Technology, 2007 (pp. 245 - 249).

[15] Lamparter, S., Oberle, D., \& Weinhardt, C. (2006). A policy framework for trading configurable goods and services in open electronic markets. ACM International Conference Proceeding Series Vol. 156: Semantic web ontologies, rules, and services track (pp. 162-173).

[16] Lauriat Shawn M., (2007), AJAX Advanced Ajax: Architecture and Best Practices, Prentice Hall PTR; 1st edition, ISBN-13: 9780131350649.

[17] Lusch, R. F, Vargo, S. L. \& O’Brien, M. (2007). Competing through service: Insights from service-dominant logic, Journal of Retailing, Vol. 83, No 1, (pp. 5-18). doi:10.1016/j.jretai. $\underline{2006.10 .002}$

[18] Lusch, R. F, Vargo, S. L. \& Wessels, G. (2008). Toward a conceptual foundation for service science: Contributions from servicedominant logic, IBM Systems Journal, Vol. 47, No. 1, (pp. 5-14). doi:10.1147/sj.471.0005

[19] Lusch, R. F. \& Vargo, S. L. (2006). Service-Dominant Logic: Reactions, Reflections and Refinements, Marketing Theory, Vol. 6, No. 3, (pp. 281-288). doi:10.1177/1470593106066781

[20] Maglio, P. \& Spohrer, J. (2008). Fundamentals of service science, Journal of the Academic Marketing Science, Vol. 36, (pp. 18-20). doi:10.1007/s11747-007-0058-9

[21] Moore, J.F. (1996). The Death of Competition: Leadership and Strategy in the Age of Business Ecosystems, J. Wiley \& Sons, Winchester, England, 1996.

[22] Musser, J. (2006). O'Reily Radar: Web 2.0 Principles and Best Practices. O’Reilly Media Inc.

[23] NESSI (2006). Strategic Research Agenda. Vol. 1. Framing the Future of the Service

[24] Normann, R. \& Ramirez, R. (1993). From value chain to value constellation: designing interactive strategy, Harvard Business Review, Vol. 71, No. 4, (pp. 65 - 77).

[25] O’Reilly, T. (2005). What is Web 2.0? Design Patterns and Business Models for the Next Generation of Software. Accessed online from http://www.oreillynet.com/pub/a/oreilly/tim/news/2005/09/ 30/what-is-web-20.html on 21 November 2008.

[26] OASIS (2006). Reference Model for Service Oriented Architecture 1.0, http://www.oasis-open.org/committees/ tc home.php? wg_abbrev=soa-rm, accessed August 2006.

[27] OASIS, (2009), Business Process Execution Language v 2.0, available at http://docs.oasis-open.org/wsbpel/2.0/OS/wsbpel-v2 .0-OS.html, retrieved 22/10/2009
[28] OASIS, (2009), Security Assertion Markup Language (SAML), available at http://www.oasis-open.org/committees/tc_home.php? wg_abbrev=security, retrieved 22/09/2009

[29] OASIS, (2009), Web Services Atomic Transaction (WSAtomicTransaction), available at http://docs.oasis-open.org/wstx/wsat/2006/06, retrieved at 22/10/2009

[30] O'Hear, S (2006). Web's second phase puts users in control. The Guardian Education. Accessed online from http://education.guardian.co.uk/elearning/story/0,,1801086,00.htm l on 21 November 2008.

[31] OMG, (2009), Business Process Model and Notation (BPMN) 1.2 , available at http://www.omg.org/spec/BPMN/1.2/, retrieved 22/10/2009

[32] Pistore, M., Traverso, P, Paolucci, M. and Wagner, M (2009). From Software Services to a Future Internet of Services, in Towards the Future Internet (eds. G. Tselentis et al.), IOS Press

[33] Prahalad, C. K. \& Ramaswamy, V. (2004). The Future of Competition: Co-Creating Unique Value with Customers, Harvard Business School Press, ISBN 978-1578519538, New York.

[34] Prahalad, C.K \& Ramaswamy, V. (2000), Co-opting customer competence, Harvard Business Review, Vol. 78, No. 1, (pp. 79 87).

[35] Prahalad, C.K and Ramaswamy, V. (2003). The new frontier of experience innovation, Sloan.

[36] Rosenberg, J. \& Remy, D. (2004). Securing Web Services with WS-Security: Demystifying WS-Security, WS-Policy, SAML, XML Signature, and XML Encryption, Pearson Higher Education, ISBN:0672326515

[37] Rust, R.T. \& Kannan, P. K. (2003). E-Service: A New Paradigm for Business in the Electronic Environment, Communications of the ACM, Vol. 46, No. 6, (pp. 37-42). doi:10.1145/777313.777336

[38] Rust, R.T. \& Lemon, K.N. (2001). E-Service and the Consumer, International Journal of Electronic Commerce, Vol. 5. No. 3, (pp. 85-101).

[39] Sawhney, M. \& Parikh, D. (2001). Where value lives in a networked world, Harvard Business Review, Vol. 79, No. 1, (pp. 79 90).

[40] Sawhney, M., Balasubramanian, S., \& Krishnan V.V. (2003) Creating growth with services, Sloan Management Review, Vol. 45, No. 2, (pp. 34-44).

[41] Spohrer, J., Vargo, S. L., Caswell, N., \& Maglio, P. P. (2008). The service system is the basic abstraction of service science. Proceedings of the 41st Annual Hawaii International Conference on System Sciences (HICSS 2008), Hawaii.

[42] Tyagi Sameer, (2006). RESTful Web Services, available at http://java.sun.com/developer/technicalArticles/WebServices/restf ul/, retrieved 22/10/2009

[43] Vargo, S.L. \& Lusch, R. F., (2004). Evolving to a New Dominant Logic for Marketing, Journal of Marketing, Vol. 68, No. 1, (pp. 117). doi:10.1509/jmkg.68.1.1.24036

[44] W3C, (2009). Resource Description Framework (RDF), available at http://www.w3.org/RDF/, retrieved 22/10/2009

[45] W3C, (2009). RDF Annotations (RDFa), available at http://www.w3.org/TR/xhtml-rdfa-primer/, retrieved 22/10/2009

[46] W3C, (2009). RDF Schema (RDFS), available at http://www.w3.org/TR/rdf-schema/, retrieved 22/10/2009

[47] W3C, (2009). Simple Object Access Protocol (SOAP), available at http://www.w3.org/TR/soap/, retrieved 22/10/2009

[48] Walters, D. \& Lancaster, G. (1999). Value and information: concepts and issues for management, Management Decision, Vol. 37, No. 8, (pp. 643- 656). doi:10.1108/00251749910291613

[49] Zuboff, S. \& Maxmin, J. (2004). The Support Economy: Why Corporations are Failing Individuals and The Next Episode of Capitalism, The Penguin Group, ISBN 978-0142003886, New York.

[50] Zongwei, L., Minhong, W., William, C., Jiming L., Tong F. \& Tan C. J. (2008). An Agent-mediated Middleware Service Framework for e-Logistics. In Eldon Y. Li \& Soe-Tsyr Yuan, Agent Systems in Electronic Business (pp. 193-217), IGI Global. 


\section{AUTHORS}

Dr Thanasis Giouvanakis holds a PhD from the University of Macedonia, Thessaloniki, Greece on "Designing and developing adaptive e-learning systems in a semantic web environment”. He also holds a Masters in Information Systems from the same university and a Diploma in Physics from the Aristotelian University, Thessaloniki, Greece.

His research interests are in the areas of e-learning, Semantic web, Software design and development and multimedia systems. He is a researcher in the Information Systems Laboratory at the University of Macedonia (team of Professor Konstantinos A. Tarabanis).

Currently he works with ALTEC on an internal project for Business Games in Immersive Environments. (email: thgiouv@uom.gr)

Dr Ioannis Ignatiadis has a $\mathrm{PhD}$ in Information Systems from the University of Bath in the UK, as well as undergraduate and MSc degrees in IT and Technology Management.

He has extensive industrial experience as a software consultant in information technology projects for large European multinationals. He also worked as a senior researcher in the implementation of EU Information Society Technologies (IST) projects, notably the LAURA FP5 and the PANDA FP6 projects. His research interests evolve around the technical, social and organizational aspects of the development and use of enterprise systems, e-business and service-oriented architecture solutions (email: jignatiadis@googlemail.com).

Adamantios Koumpis heads the Research Programmes Division of ALTEC S.A., which he founded at 1996 (then as independent division of Unisoft S.A.). Adamantios holds a $\mathrm{PhD}$ degree from the University of Kingston, UK and a Bachelor degree from the University of Crete, Greece. (email: akou@altec.gr).

Garyfallos Fragidis is Lecturer of Business Information Systems at the Department of Business Administration. He currently teaches E-commerce, Management Information Systems and Database Analysis and Design. He is the head of the Entrepreneurship Centre of the Technological Education Institute of Serres. His research is mostly related to the strategic and organizational aspects of electronic business. His current research focuses on service models for value co-creation and customer-centric aspects of electronic business. In the past he was engaged in research related to e-business models, e-services, business ecosystems, e-government and knowledge management. (email: garyf@teiser.gr).

Submitted September, $15^{\text {th }}$, 2010. Published as resubmitted by the authors on November $22^{\text {nd }}, 2010$. 